\title{
Evaluation of Simplified Compartmental Models of Reconstructed Neocortical Neurons for Use in Large- Scale Simulations of Biological Neural Networks
}

\author{
MARK E. JACKSON ${ }^{1}$ AND LAWRENCE J. CAULLER \\ School of Human Development, Program in Neuroscience and Cognition, University of Texas at Dallas, \\ Box 83068, GR 41, Richardson, TX 75803-0688, USA
}

[Received 25 March 1996; Accepted 22 October 1996]

\begin{abstract}
The electrotonic properties of the complex arborizations of neurons can be simulated by creating compartmental models based on the morphology of real neurons. These models can be very detailed with thousands of individual compartments and active channels. Large numbers of these models can be linked together into biologically realistic, large-scale neural networks with which to obtain a better understanding of the interactions among real neurons. However, the use of detailed compartmental models in such large networks is hindered by long computation times. Methods exist to reduce the complex morphology of detailed compartmental models to simpler reconstructions that retain many of the electrotonic properties of the original model yet are computationally efficient. However, little work exists that evaluates the limitations and performance of such reduced models with realistic active conductances modeled in both the soma and the dendrites to ensure that they are appropriate for use in biologically realistic network models. We have created detailed and reduced models of reconstructed dye-filled neurons from rat somatosensory neocortex and evaluated the ability of the reduced models to faithfully reproduce the input-output functions of the more detailed models. We find that the reduced models are not capable of perfectly reproducing the exact output of the detailed models using identical parameters. However, if the parameters are adjusted the reduced models are certainly capable of providing input-output patterns that are well within an acceptable range of known neural activity. The limitations and the benefits of such models are discussed. (c) 1997 Elsevier Science Inc.
\end{abstract}

KEY WORDS: Computational models, Biological neural networks, Neuronal morphology, Simplified models, Neocortex.

\section{INTRODUCTION}

The direct measurement of many of the physical properties of neurons is beyond the capabilities of the electrophysiologist. For example, while the electrophysiologist can measure the input resistance and time constant of a neuron, direct measurement of the passive membrane or intracellular resistivity is not possible. However, if the detailed morphology of the cell is known, it is possible to abstract the cell structure into a series of equivalent electrical circuits known as the cable equation [29]. This allows the complex structure of the dendrites to be converted into a set of discrete compartments. These compartmental models have provided great insight into the intricate details of single neurons and revolutionized the field of computational neuroscience. One of the greatest potentials of computational neuroscience is the use of realistic compartmental models of single neurons in largescale biologically realistic neural networks. This would allow the experimenter to simultaneously observe both the large-scale and small-scale properties of complex network behavior, something that is not technically feasible in real brain tissue.

Recent models based on morphological data contain thousands of compartments and many types of active channels $[3,34,39,40]$. Each compartment requires the calculation of several differential equations at each integration time step, which is typically on the order of $0.1 \mathrm{~ms}$. Therefore, the simulation of only a few seconds with these complex, multicompartment models requires an enormous expenditure of computational time. Thus, the use of these extremely detailed models in large-scale networks are greatly hindered by extremely long computational times. It would be of great benefit to create models with fewer compartments that retain the features of the detailed model yet are reduced to the point where they are computationally efficient. Even though the speed of modern computers is dramatically increasing, the use of reduced models will always allow the creation of larger networks using many more neuron models.

Several methods have been developed to create reduced representations of detailed compartmental models. One of the first methods was Rall's 'equivalent cylinder' representation of the motoneuron dendritic tree [29]. Several conditions must be met to make this reduction valid [29,30,32], among them:

1. The electrical properties of the neuronal membrane must be uniform over all dendrites.

2. All terminal branches must end with the same distal boundary condition (usually sealed end condition).

3. All terminal branches must end at the same electrotonic distance from the origin.

4. At every branch point, the sum of the diameters of the daughter branches raised to the $3 / 2$ power must equal the $3 / 2$ power of the parent branch diameter (Rall's 3/2 Power Rule). This

\footnotetext{
${ }^{1}$ To whom requests for reprints should be addressed.
} 
ensures impedance matching by conserving the product of the membrane resistivity $\left(R_{m}\right)$ to the intracellular resistivity $\left(R_{a}\right)$.

If these conditions hold for a particular dendritic tree, then that tree can be reduced into an equivalent cylinder whose diameter is calculated as follows:

$$
d_{e q}^{3 / 2}=\sum_{j=1}^{n} d_{j}^{3 / 2}
$$

where $d_{\mathrm{eq}}$ is the diameter of the equivalent cylinder and $d_{\mathrm{j}}$ is the diameter of each of the $\mathrm{n}$ dendritic branches to be reduced. The first two criteria are usually assumed to hold but, at least for neocortical cells, few dendritic arborizations meet the last two criteria [24].

An alternative method that copes with arbitrary geometries transforms the dendrites into normalized, electrotonically equivalent lengths [14,31,35], which are then combined using the $3 /$ 2 Power Rule to produce an electrotonically equivalent profile. By varying its diameter along its length, this single profile averages out variations in the dendritic structure yet retains many of the electrotonic properties of the more complex system of branched dendrites. Stratford et al. [35] improved on this method to create a cartoon representation of a pyramidal neuron that separated the basal and apical domains and included several lumped side branches along the apical trunk to represent the apical oblique branches. Voltage transients obtained from this cartoon were superior to those obtained from simple single equivalent cylinder representations.

Bush and Sejnowski [7] developed an alternative reduction method that conserves the axial resistance rather than the surface area of the dendritic tree. This is done by making the crosssectional area of the equivalent cylinder equal to the sum of the cross-sectional areas of all the dendrites represented by that equivalent cylinder. The length of the equivalent cylinder is the average length of the dendrites that are represented by the equivalent cylinder. Because the surface area is not conserved, $R_{m}$ and $C_{m}$ must be scaled for each equivalent cylinder to match the $R_{\text {in }}$ and $\tau_{\mathrm{m}}$ of the detailed model. The authors consider the advantages of this method to be that it is simpler than the cartooning procedure of Stratford et al. [35] and the lengths of the equivalent cylinders are equal to the average length of the dendrites they represent. This may make it easier to specify spatial connections in a network.

A more unique method of neuron reduction was developed by Brown and Zador [6]. They determined the voltage response due to synaptic current at every point in the dendritic tree, and formed a look-up table that could be referred to to determine the effect of any particular synaptic input. Although this obviously requires a great amount of setup time, because every possible response must first be simulated so it can be added to the table, during the actual simulation run the look-up table is much more efficient than calculating the result of thousands of differential equations. To help interpret the massive amount of data that is produced by the simulation run, they developed a graphical method of representation, a morphoelectronic transform that preserves the diameter and branching pattern of the dendrites but substitutes a measure of the electrotonic length for the anatomical length. Besides computational efficiency, this method has the advantage of preserving the spatial integration of synaptic inputs because the dendritic tree is still represented in detail. The disadvantage of this method is that temporal integration of synaptic inputs is not represented unless phase-delay or time-to-peak are substituted for the electrotonic length dimension, so spatial and temporal aspects of synaptic integration cannot be explored si- multaneously. Also it is not convenient to add the effect of nonlinear dendritic conductances. While this method is quite efficient for examining the effect of synaptic integration in a passive neuron, it ultimately is not well suited to exploring the network behavior of neurons with complex active dendritic conductances.

The reduction methods by both Stratford et al. [35] and by Bush and Sejnowski [7] have been used to create reduced models of neocortical neurons that accurately reproduce the electrotonic properties of the more detailed multicompartmental models they represent. However, it is not clear how well these reduced models compare to the detailed models when realistic, active conductances are modeled in both the soma and the dendrites. Before reduced models can be used in biologically realistic large-scale networks, it is crucial to first evaluate their limitations and capabilities.

Toward this end, we have morphologically and physiologically identified numerous neocortical neurons in the somatosensory cortex of rats by intracellular injection of biocytin and created multicompartment neuron models. Specifically, we have detailed models of three major neural subtypes; layer III regularspiking (L3) and layer V bursting pyramidal (L5) neurons with extensive apical tufts, and layer IV fast-spiking spiny stellate (L4) neurons. We developed reduced models of these cells with a slight modification of the method of Stratford et al. [35]. We emphasize an evaluation of the ability of the simplified models to faithfully reproduce the passive membrane properties of the detailed models as well their input-output functions with realistic active conductances incorporated. Our goal is to evaluate if the reduced models can be expected to provide reasonable representations of the behavior of more detailed cell models when used in large-scale networks. Preliminary descriptions of this work were presented elsewhere [16].

\section{MATERIALS AND METHODS}

\section{Development of Compartmental Models}

Coronal slices ( $400-450 \mu \mathrm{m}$ thick) of SI neocortex were prepared from adult (100-450 g) male and female, albino and Long-Evans rats (Charles River) with a vibratome in the usual way [12]. The slices were maintained in vitro in a Haas-type interface chamber (Medical Systems) with perfusion ( 0.5 to 1 $\mathrm{ml} / \mathrm{min}$.) of artificial cerebrospinal fluid (in mM: $\mathrm{NaCl} 126, \mathrm{KCl}$ $3, \mathrm{MgSO}_{4} 1-2, \mathrm{CaCl}_{2} 2, \mathrm{NaHCO}_{3} 26, \mathrm{NaH}_{2} \mathrm{PO}_{4} 1.25$, dextrose 10; saturated with $95 \% \mathrm{O}_{2}, 5 \% \mathrm{CO}_{2}, 7.4 \mathrm{pH}, 31-34^{\circ} \mathrm{C}$ ). The slices were prepared from the middle third of the cerebral hemisphere from the rostral septum to the caudal striatum. Care was taken to section the slice perpendicular with respect to the midsagittal and dorsal planes to maintain continuity of the apical dendrites of deep pyramidal neurons. Only cells whose dendrites were completely contained within the thickness of the slice were analyzed for this study.

Sharp micropipettes (Brown Flaming puller, 120-160 M $\Omega$ ) filled with $1 \mathrm{M} \mathrm{K}$-acetate $(7.4 \mathrm{pH})$ were used for intracellular penetrations and $\mathrm{R}_{\mathrm{in}}$ and $\tau_{\mathrm{m}}$ were measured for each cell. Intracellular recording and current injection were controlled with a current clamp electrometer (Axoclamp-2A, Axon Instruments). To stain penetrated cells, biocytin (3-5\%; Molecular Probes) was added to the filling solution and injected by electrophoresis ( $0.5 \mathrm{nA}, 50 \%$ duty cycle, $1 \mathrm{~Hz}$ for $10 \mathrm{~min}$ ). Slices were fixed by submersion overnight in $4 \%$ paraformaldehyde phosphate buffer (PF; $0.1 \mathrm{M}, 7.4 \mathrm{pH}$ ), then they were submerged in $30 \%$ sucrose phosphate buffer until they sank before they were frozen sectioned, $60 \mu \mathrm{m}$ thick through the plane of the slice (i.e., 6-7 sections/slice). Following $\mathrm{H}_{2} \mathrm{O}_{2}(0.5 \%)$ and Triton X-100 (1\%) pretreatment, sections were processed for biocytin by overnight 
incubation in avidin-HRP ( $\mathrm{ABC}$ standard, Vector Labs). Then the HRP was reacted with cobalt-intensified DAB (protocol available upon request).

The morphologies of biocytin-filled neurons were reconstructed in three dimensions by means of computer-assisted camera Lucida tracing (NeuroLucida by Jack Glaser, MicroBrightfield) under $100 \times$ oil immersion [8,10]. The camera Lucida drawings of the three neurons used in this study are shown in the first column of Fig. 1. The measured lengths and diameters of these neurons were used to establish the parameters of equivalent electrical circuits using NEURON [13], and schematic diagrams of the compartmental models are shown in the middle column of Fig. 1.

A geometric adjustment was made to all compartmental segments to account for the fact that morphological measurements of the original neuron may have been underestimated due to either shrinkage of the brain tissue during histological processing or due to optical distortions during measurement. Estimates of tissue shrinkage were based upon comparison of distances between landmarks measured in vitro with measurements obtained after histological processing. We observed significantly more shrinkage in the thickness of the slice and sections than within the plane of the sections. A shrinkage factor of $10 \%$, based upon the average of shrinkage observed in all dimensions across all slices, was applied to the length and diameter of all compartments in accordance with previous simulations $[8,35]$.

The model parameters were also adjusted to account for the surface area contributed by spines. The thousands of dendritic spines present on neocortical pyramidal neurons greatly increase the membrane surface area [22,23]. Explicit modeling of spines requires thousands of additional compartments and is, therefore, computationally expensive. Spines are routinely implicitly added to compartmental models [31] by increasing compartmental $\mathrm{C}_{\mathrm{m}}$ and reducing $\mathrm{R}_{\mathrm{m}}$ according to the proportion of the total membrane area due to spines. We added $1.25 \mu \mathrm{m}^{2}$ of surface area per $\mu / \mathrm{m}$ of dendritic length (average spine density $\times$ average spine area $=1.3 \times 0.96)$ as in Cauller and Connors [8] .

\section{Simulations}

Simulations were performed using both NEURON [13] and GENESIS [4,42] running under UNIX on a SUN workstation. Both simulation packages are available in the public domain. Analysis of the models using either simulation package produced comparable results. Most of the data that we report here were generated using NEURON. The simulations involving calcium conductances were run in GENESIS using channels adapted from the Traub91 [40] of channels. The integration time step in all instances was $0.01 \mathrm{~ms}$.

To make a consistent set of comparisons across each model, a uniform set of passive membrane parameters were used for each model. As is described below in detail, $R_{m}$ and $C_{m}$ were adjusted in the reduced models to more closely match certain aspects of the detailed model. The passive membrane parameters were selected for their biological relevance. Homogeneously distributed membrane $\left(\mathrm{C}_{\mathrm{m}}\right)$ was set at the commonly used value of capacitance $1 \mu \mathrm{F} / \mathrm{cm}^{2}$ [11]. Membrane resistivity $\left(\mathrm{R}_{\mathrm{m}}\right)$ and intracellular resistivity $\left(R_{a}\right)$ parameters were set at the values we obtained from matching the detailed cell models to the charging curves obtained in vitro for the cells upon which the models are based. For this purpose we assumed a somatic leak from the sharp electrode and found that the charging curve, membrane time constant $\left(\tau_{\mathrm{m}}\right)$, and the input resistance $\left(\mathrm{R}_{\mathrm{in}}\right)$ could not be matched to the physiological data with $\mathrm{R}_{\mathrm{a}}<200 \Omega$-cm. At this value of $\mathrm{R}_{\mathrm{a}}$ we found that the cell models matched the in vitro data if $R_{m}$ was

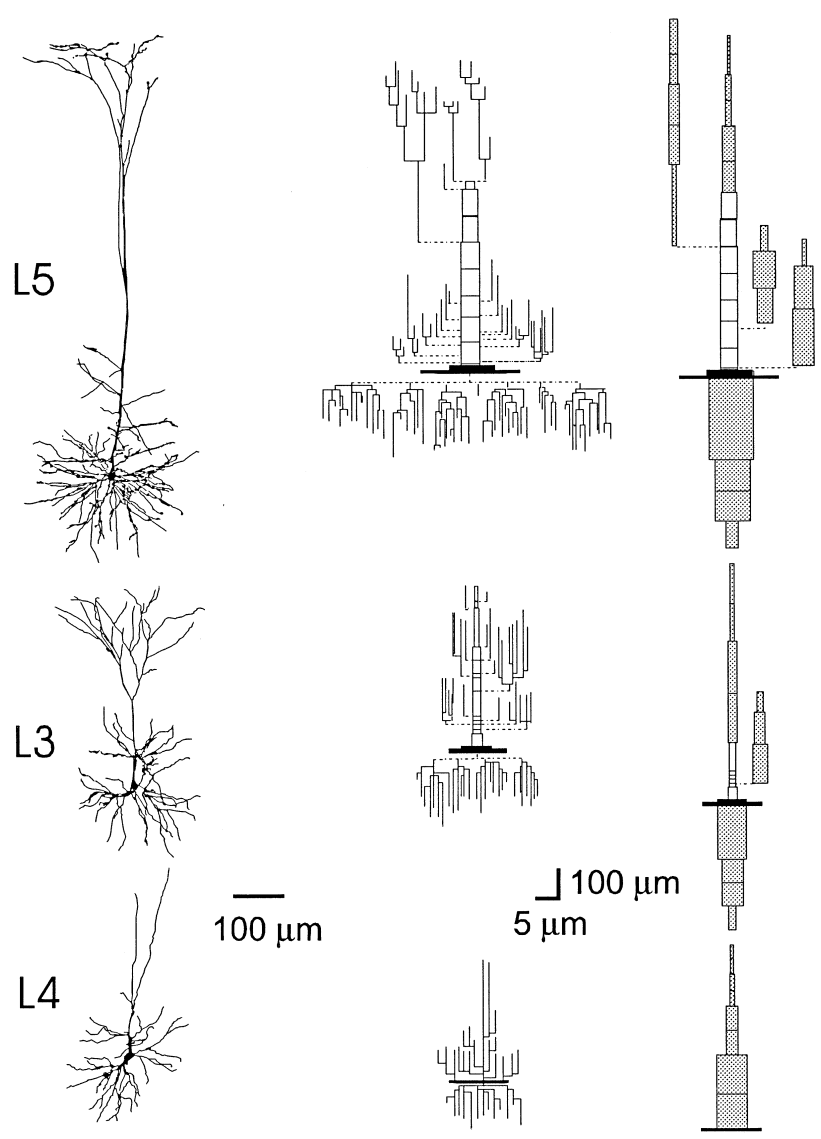

FIG. 1. (Left) Camera-Lucida drawings of three biocytin-filled neocortical neurons. Cell types are, from top to bottom, layer V bursting pyramidal (L5), layer III pyramidal (L3), and layer IV spiny stellate ( star pyramid) (L4). Scale bar is on the left. (Center) Schematic drawings of the compartmental models representing the neurons at the left. The length of each dendrite is drawn to scale to illustrate the general morphology patterns. Individual diameters are not drawn to scale, with the exception of the apical trunk and soma. (Right) Scaled drawings of the reduced compartmental models. Reduced sections are shown as shaded regions and unreduced sections are shown as open or black (soma).

around $10,000 \Omega-\mathrm{cm}^{2}$. Based on these results, $R_{a}$ and $R_{m}$ were set at homogeneously distributed values of $200 \Omega-\mathrm{cm}$ and 10,000 $\Omega-\mathrm{cm}^{2}$, respectively. These values were used in most of the simulations reported here. The somatic leak was only used when the model was matched to the physiological data for the purpose of estimating realistic values for $R_{m}$ and $R_{a}$; all other simulations assumed homogeneous membrane parameters because the goal of this work is to evaluate neuronal function in the absence of intracellular electrodes. We also tested the models using a range of realistic parameters (i.e., $\mathrm{R}_{\mathrm{m}}=100,000 \Omega-\mathrm{cm}^{2}, \mathrm{R}_{\mathrm{a}}=500 \Omega$ $\mathrm{cm})$ and data from some of those simulations are also reported.

\section{Measurement of Simulated Input Resistance and Time Constant}

The input resistances $\left(\mathrm{R}_{\text {in }}\right)$ of the modeled neurons were measured by simulating a continuous step injection of a known amount of hyperpolarizing current and measuring the resulting steady-state change in the membrane potential. The membrane time constant $\left(\tau_{\mathrm{m}}\right)$ was measured as the inverse negative slope of the linear portion of the natural log-linear charging curve measured between $5 \mathrm{~ms}$ and $10 \mathrm{~ms}$ in response to an extended hy- 
perpolarizing current pulse. Only passive, electrotonic properties were simulated during these measurements.

\section{Development of Reduced Models}

These morphologically detailed compartmental models were transformed into simplified, computationally efficient models following the methods described in Stratford et al. [35]. Briefly, dendritic trees that terminated at approximately equal electrotonic distances from the soma were collapsed into equivalent profiles. These equivalent profiles were then smoothed into a series of cylindrical compartments with a diameter equal to the average diameter of the equivalent profile. The electrotonic length of each compartment was not allowed to exceed $0.1 \lambda$ to ensure accurate computation of transients [31]. For the pyramidal cell models, the basal, apical, and oblique dendrites were reduced separately, keeping the apical trunk unreduced. We found that this cartoon provided the best reproduction of passive properties for the pyramidal cells, in agreement with Stratford et al. [35].

We found that we could improve the performance of our models by slightly modifying the method of Stratford et al. [35]. The reduction of the dendrites to an equivalent profile results in a profile that varies in diameter along its length in steps of $1 \mu \mathrm{m}$ that preserves the original surface area. This profile is then smoothed into a series of cylindrical compartments. Because this smoothing process required an averaging of the diameters of the equivalent profile, the surface area is not perfectly preserved and $\mathbf{R}_{\text {in }}$ and $\tau_{\mathrm{m}}$ are not perfectly matched. We found that we could correct for this small error by optimizing the performance of each section as it was reduced. This was accomplished by first adjusting $R_{m}$ for the reduced cylinders to match the original somatic $\mathrm{R}_{\text {in }}$. Then charging curves were obtained for both the detailed and the reduced models and the normalized simple root mean squared (RMS) difference between the curves was measured. $\mathrm{C}_{m}$ was then adjusted for the reduced cylinders to minimize the RMS difference. Thus, each reduced section had an adjustment factor for $\mathrm{R}_{\mathrm{m}}$ and $\mathrm{C}_{\mathrm{m}}$ that provided the best possible match to the charging curve of the detailed cell model. This results in a small increase in the overhead of implementing the model, but these adjustments are easily made in both NEURON and GENESIS when the cell description file is read in and the resulting increase in performance of the models is well worth the small effort.

Because we wanted to make models with a minimum number of compartments to aid in computational efficiency, we reduced the oblique dendrites to as few sections as possible while retaining a reasonable level of electrotonic fidelity. All of the L3 cell apical oblique dendrites happened to terminate at close to the same electrotonic distance from the soma, so they were reduced into a single equivalent profile. However, reducing all of the oblique dendrites of the much larger L5 cell into a single equivalent profile made it very difficult to match the somatic response of the reduced model to the detailed model when current inputs were applied to the distal apical trunk. The morphology of the L5 cell suggested two distinct apical oblique domains, a proximal section with extensively branched, compactly spaced dendrites, and a more distal section with unbranched, spatially dispersed dendrites. Therefore, two sections of oblique equivalent profiles were produced. The optimum placement along the apical trunk of the most proximal of these was determined by comparing the RMS difference between charging curves recorded in the soma of the detailed and a partially reduced model, with only the proximal obliques reduced and the distal obliques modeled in full. The position of the obliques was adjusted to minimize the RMS difference between the charging curves of the two models. The position of the distal oblique section was then optimized to give the best response to a current stimulation applied at the apical nexus, the major branch point on the distal apical trunk [8] (arrow on abscissa of Fig. 2). The reduced distal oblique section was systematically positioned at different locations along the apical trunk. Current was applied at the apical nexus and $R_{\text {in }}$ was measured at several locations along the apical trunk. Figure 2 shows the accuracy, measured as the percent difference of the $\mathrm{R}_{\mathrm{in}}$ measured at several identical positions along the apical trunk in the detailed and the reduced models, for three different positions (open squares) of the distal oblique section. The thick line in Fig. 2 shows the optimum position of the distal oblique section, which minimizes the difference, while the dashed and dotted lines show the effects of moving the distal oblique section slightly more proximal and slightly more distal to the soma, respectively. $R_{\text {in }}$ is not well matched along the entire length of the apical trunk because the distribution of the impedance load provided by the oblique dendrites has changed.

The results of these transformations for each of the three cell types are shown in the right column of Fig. 1. The reduction process for the three cell models resulted in a significant reduction in the number of simulated compartments. The L 3 cell model was reduced from 217 compartments to 32 compartments, the L4 cell model was reduced from 156 compartments to 12 compartments, and the L5 cell model was reduced from 710 compartments to 50 compartments.

\section{RESULTS}

Figure $3 \mathrm{~A}$ shows the somatic charging curves for the detailed and reduced versions of each cell model in response to a $50 \mathrm{~ms}$ depolarizing somatic current injection and subsequent discharge. The cell models contained only passive properties. Recordings are also shown for the two pyramidal cell models in response to current injection in the distal apical trunk. The solid lines represent the detailed models and dashed lines represent the reduced models. In each case the steady state charging curves for the detailed and reduced models are almost indistinguishable.

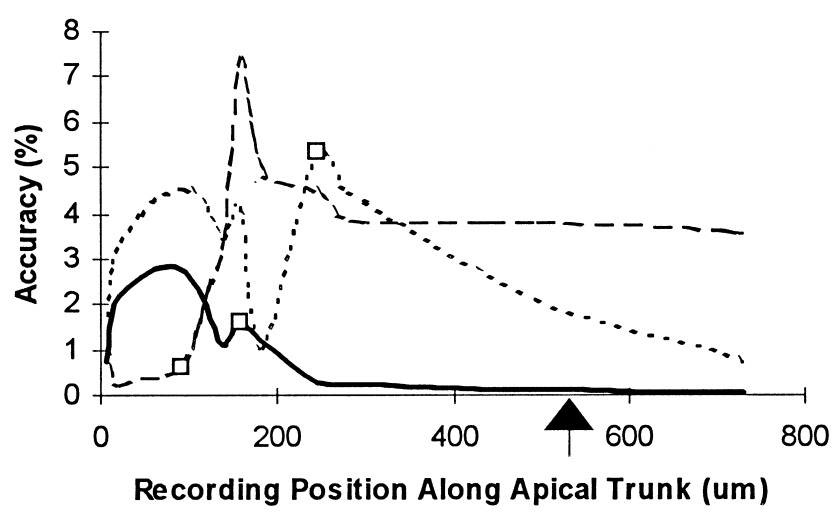

FIG. 2. Effect of the position of the distal oblique section on the local charging curves recorded in the apical trunk of the layer V cell model. $\mathrm{R}_{\text {in }}$ was recorded at various points along the apical trunk of the detailed compartmental model and compared to values obtained along the apical trunk of the reduced model with the reduced distal oblique section placed at various locations along the trunk. The solid line shows the position at which a minimum difference was seen at the distal apical trunk. The dashed line shows the effect of moving the distal oblique section more proximal to the soma and the dotted line shows the effect of moving the distal oblique section more distal to the soma. The position of the distal oblique section is indicated by an open square superimposed on the respective line. 


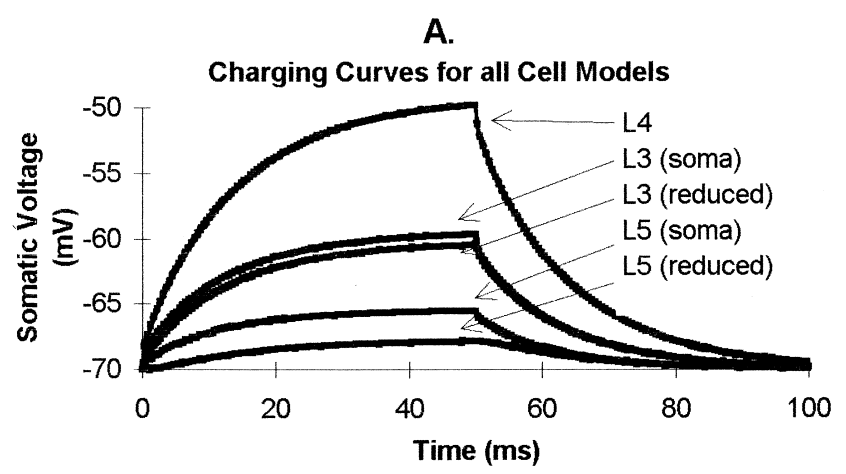

B.

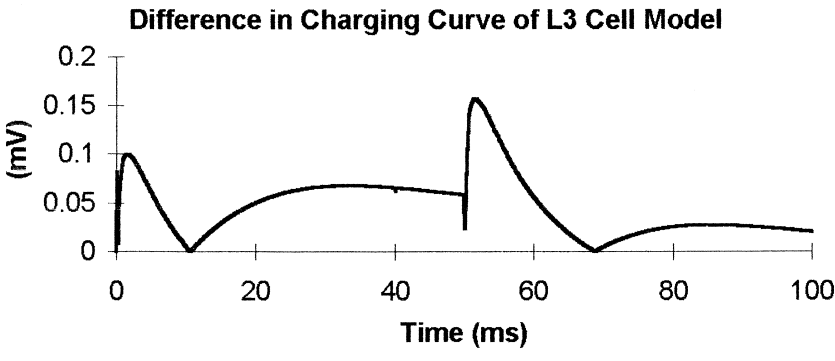

C.

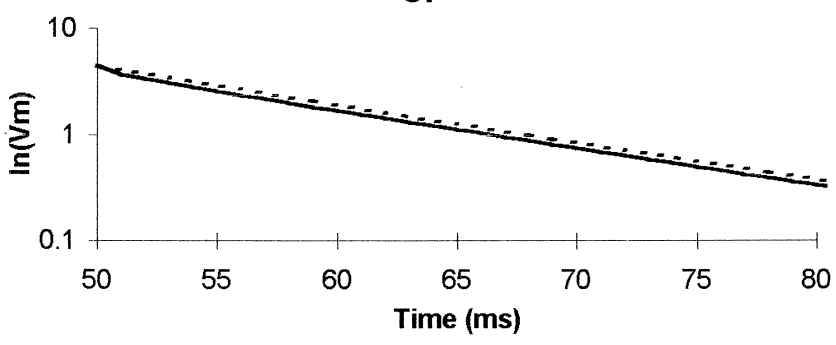

FIG. 3. (A) Charging and discharging curves for each cell model in response to a $50 \mathrm{~ms}$ current injection in both the soma and, for the two pyramidal neurons, at the most distal apical trunk segment. The solid lines represent the detailed compartmental models and the dashed lines represent the reduced models. Note that the curves for the detailed and the reduced models are almost superimposed upon each other. (B) Difference between the charging curves of the L3 detailed and reduced models. The greatest difference is seen at times soon after the voltage transients. This reflects the incomplete matching of the early time constants by the reduced model. (C) Log plot of the discharging portion of (A) showing that the late time constant is the same for both models (identical slopes), but the early time constants are not matched by the reduced model (offset of lines).

However, closer inspection reveals a small but possibly significant difference between the charging curves for the two models. A typical example is seen in Fig. 3B, which shows the difference between the charging curves shown in Fig. 3A for the L3 cell models with somatic current injection. In general, the difference between the two charging curves appears to be small, with the maximum percent difference $<1.5 \%$ (mean $0.7 \%, \mathrm{SD}$ $0.66 \%$ ). The difference was a minimum at times close to $\tau_{\mathrm{m}}$, not surprising because $\mathrm{C}_{\mathrm{m}}$ for each reduced section was adjusted to match $\tau_{\mathrm{m}}$ of the detailed model. However, an exact matching of the charging curves for the detailed and reduced models was not possible because all of the early time constants could not be matched by the reduced models. The membrane time constant $\left(\tau_{\mathrm{m}}\right)$ is but one of many time constants associated with a particular neuronal morphology $\left(\tau_{n}, n \geq 0\right)$. In our case, because we defined $R_{m}$ to be uniform everywhere in the cell, $\tau_{0}=\tau_{m}$. Typically, $\tau_{\mathrm{m}}$ is measured after the voltages in all parts of the cell have equalized following an initial voltage transient. As the voltage response to a current injection spreads through the dendrites, each section of dendrite contributes its own small portion to the sum of all $\tau_{n}(n>0)$. For a compartmental neuron model, there is a $\tau_{n}$ associated with each separate compartment. By definition, when the detailed model is reduced to a fewer number of compartments there is a loss in the number and magnitude of $\tau n$. Rall et al. [31] illustrated this point very well by comparing the peeled time constants of a branched tree (143 compartments) to an equivalent cable representation of the same tree (38 compartments ). While $\mathrm{R}_{\text {in }}$ and $\tau_{0}$ were identical in both cases, $\tau_{n}$ for $n$ $>0$ were several orders of magnitude lower for the equivalent cylinder.

The effect of this incomplete matching of $\tau n$ by the reduced model is seen in Fig. 3B. The greatest difference in the charging curves is seen soon after the current injection begins $(0 \mathrm{~ms})$ and stops $(50 \mathrm{~ms}$ ). While still not large, these early differences may become significant when active channels with nonlinear voltage dependencies are added to the models. This effect is also seen in Fig. 3C, which shows that $\tau_{\mathrm{m}}$ of the L5 cell model, measured as the inverse slope of the natural log of the discharge curve at late time intervals, is the same for both the detailed and the reduced models, because the slope of the line is identical at late time intervals. However, the two lines are offset because the early time constants are different. This may be particularly troublesome because the greatest difference occurs so early $(<2 \mathrm{~ms})$ after the current transient. Because this is within the time frame of a typical action potential, it is possible that this small difference in the charging curves may have a great effect on the timing of action potentials, and this error may propagate with subsequent action potentials.

More insight into the electrotonic filtering properties of the reduced models can be gained by comparing their responses to rapidly changing inputs. Figure $4 \mathrm{~A}$ is a plot of the steady-state somatic input impedance of all models following a sinusoidal (AC) current injection at the soma over a wide range of stimulation frequencies. AC analysis allows an exploration of both the capacitive and resistive components of the input impedance. At low frequencies the capacitive component will be highest and the flow of current in the circuit will be mostly through the membrane resistance, while at high frequencies the capacitive impedance will fall and current will bypass the membrane resistance. Biological membranes have the features of a low-pass filter and faithful reproduction of this feature is essential for a model to reflect accurately the input-output properties of real neurons. Although this data has not often been measured for real neurons because of the confounding effect of voltage-dependent conductances, it is a well established property of nerve membrane $[11,15]$.

In Figure 4A, the $\mathrm{AC}$ impedance spectrums for the detailed (solid lines) and reduced models (dashed lines) appear to be practically identical for each of the three cell models and are typical of a low-pass filter. The impedance drops rapidly as the stimulation frequency increases, with a steep roll-off above 10 $\mathrm{Hz}$, as is typical of real neuron membrane [15]. Figure 4B shows the percent difference between the sets of curves in Fig. 4A. The difference between the detailed and the reduced models is very small at low frequencies, but the differences grow as the stimulation frequencies increase above $30 \mathrm{~Hz}$. The two reduced pyramidal cell models (L3 and L5) have impedances as much as 

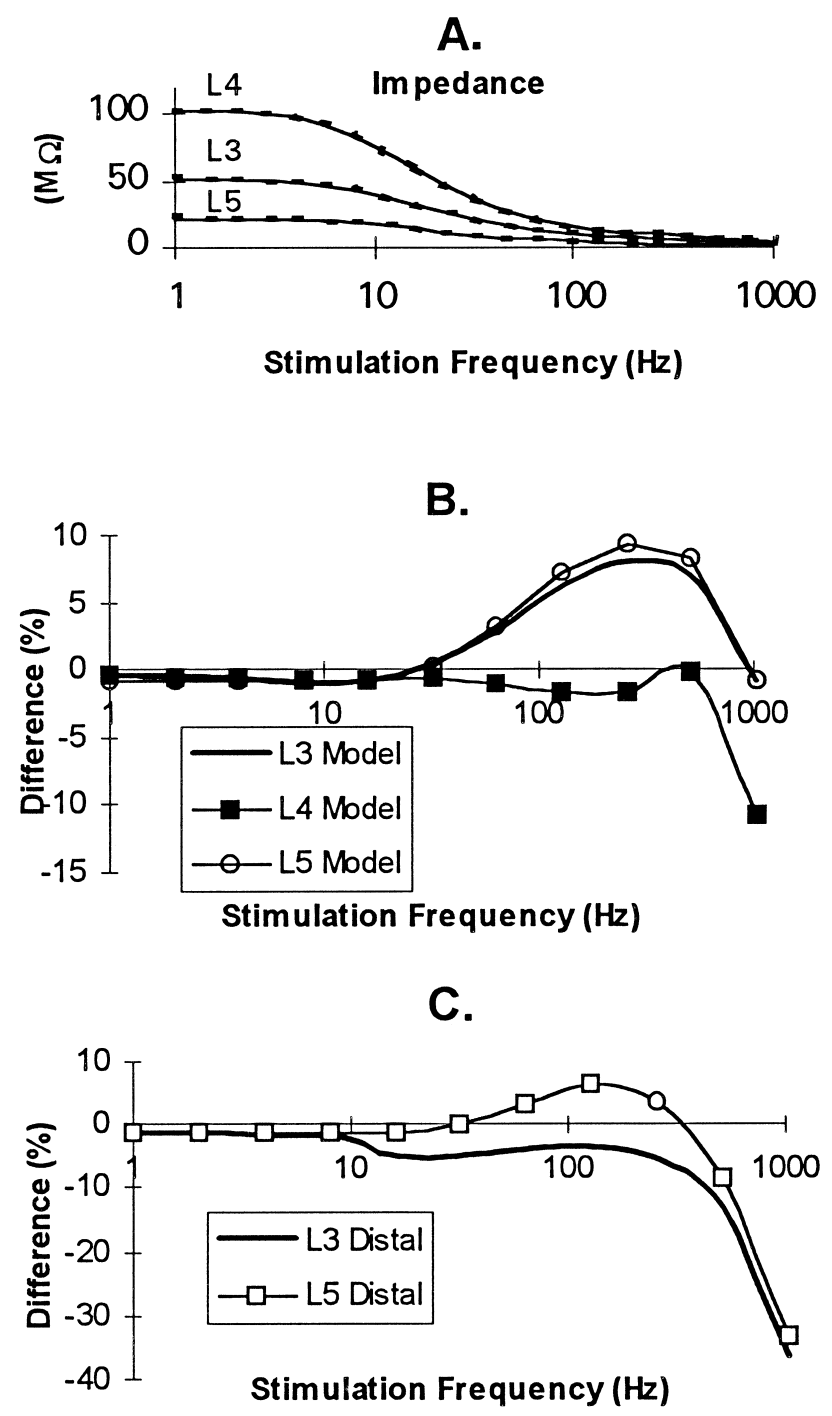

FIG. 4. (A) Plot of the somatic input impedance following sinusoidal (AC) current injection of various frequencies at the soma. The solid lines are the detailed compartmental models and the dashed lines are the reduced models. Note the very close matching of the detailed and reduced models. (B) Difference in the curves in (A) between the detailed and reduced models expressed as a percent. At low frequencies the differences are minor but the differences increase at higher input frequencies. (C) Same as (B) except the stimulation current is applied to the distal apical trunk of the two pyramidal cell models. Note the difference in the scale.

$10 \%$ higher than the respective detailed cell models at $256 \mathrm{~Hz}$. The reduced L4 model is closely matched to the impedance of the detailed cell model at frequencies up to $512 \mathrm{~Hz}$, but it quickly diverges as the stimulation frequency reaches $1024 \mathrm{~Hz}$. Figure 4C shows the same data as Fig. 4B except that the sinusoidal stimulation current has been applied to the distal apical trunk of the two pyramidal cell models. In this stimulation condition, the reduced L3 cell model has a higher than normal impedance for frequencies above $32 \mathrm{~Hz}$ but the impedance rapidly drops above $256 \mathrm{~Hz}$, with the difference exceeding $30 \%$ at $1024 \mathrm{~Hz}$. The impedance of the reduced $\mathrm{L} 4$ cell model is always lower than the detailed L4 cell model and exhibits the same rapid decrease above $256 \mathrm{~Hz}$.
It is apparent that the reduced models do not faithfully reproduce the detailed model's response to high-frequency inputs and, therefore, the low-pass filtering properties of the reduced models is different. For the two reduced pyramidal cells, the impedance is greater than that for the detailed models in a range of input frequencies from 60 to $512 \mathrm{~Hz}$. Thus, the reduced models might have a greater voltage response to inputs within this range of frequencies. This might be an even greater factor when active channels are added to the passive membrane.

As a final test of the passive membrane properties of the reduced models, we determined the somatic responses to various synaptic inputs. Specifically, we wanted to know if synaptic inputs into the reduced basal or apical tuft sections could accurately reflect a similar synaptic input in the same general dendritic areas of the detailed model. Figure 5 compares the somatic excitatory post synaptic potentials (EPSPs) for the L5 cell detailed and reduced models. Synaptic inputs were simulated by an alpha function $\left(\tau_{\text {alpha }}=1 \mathrm{~ms}\right)$. The dashes represent the separate responses to unitary synaptic inputs $(1 \mathrm{nS})$ applied to each basal dendrite compartment in the detailed model. The open circles represent the somatic responses to the same synaptic inputs applied to each basal compartment of the reduced model. These plots represent the characteristic range of synaptic EPSPs of each model, showing the possible range of peak amplitudes and timeto-peak for simple synaptic inputs. The range of responses of the reduced model fall outside of the range of the possible responses of the detailed model using the same synaptic parameters.

This finding raised an interesting point. Many of the parameters that were being used to test the models were essentially educated guesses that were picked from within a range of possible values. Apparently, the reduced models could not reproduce the detailed behavior of the detailed cell models if identical parameters were used. However, there is no reason to believe that the parameters used in the detailed model are either unique or correct. What is of most interest here is whether the reduced models can behave the same way as the detailed models in response to inputs, within a reasonable range of parameters. For example, the parameters used to simulate the synaptic inputs to the detailed cell model were based on some generally accepted parameters. However, a fairly wide range is possible for many of those parameters. Therefore, we attempted to match the EPSPs

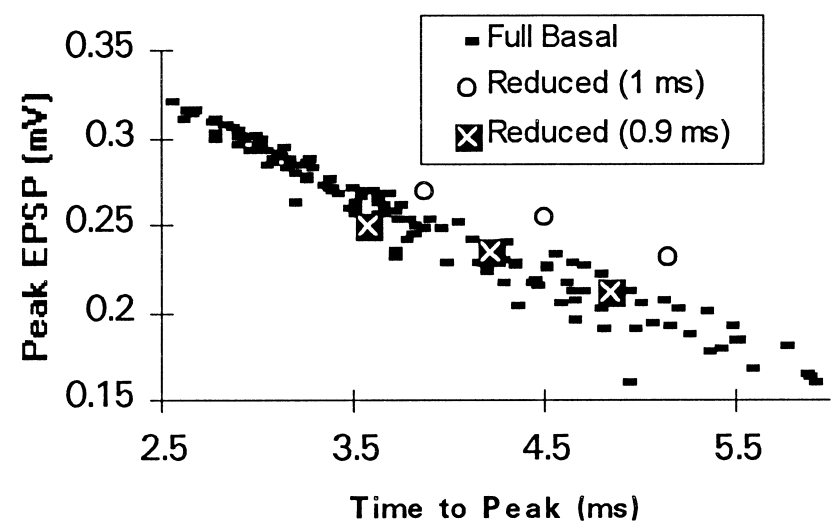

FIG. 5. Plot of EPSP's recorded in the soma of the layer V cell model. Each dash represents the time to peak and peak amplitude of the EPSP produced by a unitary synaptic input into a single basal dendritic compartment of the detailed model. The open circles represent the reduced dendritic sections. The crossed circles show the reduced dendritic responses following adjustment of the synaptic alpha function time constant from $1.0 \mathrm{~ms}$ to $0.9 \mathrm{~ms}$. 
of the reduced models to the characteristic range of EPSPs of the detailed models by adjusting some parameters within the range of biological possibility. We found that if we changed $\tau_{\text {alpha }}$ from $1.0 \mathrm{~ms}$ to $0.9 \mathrm{~ms}$, the EPSPs in the reduced model (solid triangles in Fig. 5) moved into the range of the EPSPs from the detailed model. Of course, because the reduced model only has a few isopotential basal compartments, it is not possible to exhibit the entire range of EPSPs evident in the detailed cell model. Similar results were obtained with apical dendrite inputs and for different synaptic magnitudes.

Next, we added active channels to each model to observe the input-output functions of active models. Standard HodgkinHuxley type sodium and potassium channels were added to the soma of each model, with identical parameters used for both the detailed and the reduced models. Figure 6A shows the action potentials produced in both the detailed and reduced models of the L5 cell following identical $1 \mathrm{nA}$ step current injections in the soma. The depolarizing current was turned on at $0 \mathrm{~ms}$ and turned off at $20 \mathrm{~ms}$. Figure $6 \mathrm{~B}$ shows the spike activity produced by a $20 \mathrm{~ms}, 5 \mathrm{nA}$ step current injection in the distal apical trunk. In both cases the reduced model (dashed lines) very closely follows the activity of the detailed model (solid lines). Note that in both cases the models fired the same number of action potentials and at very nearly the same frequency. Similar results were obtained for the L3 and L4 models. Thus, despite the fact that the reduced models imperfectly matched the passive properties of the detailed models as detailed above, little effect is seen in the input-output function of active channels in the soma, at least with very simple voltage-dependent active channels.

To further explore the input-output functions of the models, an A-type potassium current was added to the standard HodgkinHuxley channels. Figure $6 \mathrm{C}$ shows a plot of the firing frequency vs. the time to first spike following increasing steady-state current injection. In all three cases the reduced models (dashed lines) closely follow the responses of the detailed models (solid lines). Small differences can be seen, but the performance of the reduced models is well within the range of expected behavior. This result was obtained without any active channel parameters being changed between the detailed or the reduced models. However, active conductances were only located in the soma.

As an even more realistic test of the input-output responses of the models, we added calcium dependent conductances together with voltage dependent conductances not only to the soma, but also throughout the dendritic tree. These simulations were run in GENESIS using the Traub91 [40] set of active channel models. For the L3 cell models, active channels were added that would produce the adapting spike train that is typical of L3 regular spiking pyramidal neurons in neocortex [1]. The channels consisted of a fast sodium $(\mathrm{Na})$, potassium delayed rectifier $(\mathrm{K}$ $\mathrm{DR})$, high-threshold calcium ( $\mathrm{Ca})$, calcium dependent potassium $\left(\mathrm{K}_{-} \mathrm{C}\right)$, A-current potassium $\left(\mathrm{K}_{-} \mathrm{A}\right)$, afterhyperpolarization calcium and voltage dependent potassium ( $\mathrm{K}_{-} \mathrm{AHP}$ ), and intercellular calcium concentration $\left(\mathrm{Ca} \_\right.$conc $)$.

At first, these channels were added only to the soma, and the channel parameters were adjusted in the detailed cell model to produce an adapting spike train in response to somatic current injection. Then the same parameters were transferred to the reduced model. The resulting comparison of spike trains is seen in Fig. 7A. While both cell models do produce adapting spike trains, the timing of spikes is very different between the detailed and the reduced model. The effect of the incomplete matching of the early time constants by the reduced model has now been revealed by the complex interactions of the nonlinear kinetics of the calcium and voltage dependent channels.
However, as discussed above, it is not necessary to require that the same channel parameters used in the detailed model should transfer directly to the reduced model. There was nothing unique about the parameters used in the detailed model; they simply worked. However, many other combinations of parameters would have also worked. Therefore, we modified the channel parameters of the reduced model to qualitatively match the output of the reduced model to that of the detailed model. However, the parameter space is quite large so we were not able to obtain an exact match, yet we did obtain qualitative matches using many sets of parameters.

The most realistic condition of active dendritic conductances proved to be problematic to implement as a test. Because the dendritic morphology of the detailed and reduced models was by definition quite different, there was no way to compare channel distributions one to one. Nonetheless, we placed $\mathrm{Na}, \mathrm{K} \_\mathrm{DR}, \mathrm{Ca}, \mathrm{K} \_\mathrm{C}$,

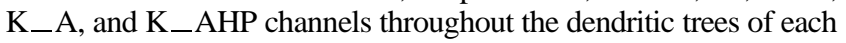
model. The channel parameters (i.e., density, maximum conductance) were adjusted for each model independently to produce representative input-output curves from each cell type (Fig. 7B-D).

Table 1 shows the time (in seconds) required to compute $1 \mathrm{~s}$ of simulated time for each model on a Sparc 20 workstation using NEURON. Each model was simulated individually with only passive membrane and also with active dendrites. The computational efficiency of the reduced models is obvious.

\section{DISCUSSION}

We conclude that reduced computational models are more efficient for use in large-scale biologically realistic neural networks, but this increased efficiency is balanced against a loss of input-output fidelity. However, while $\mathbf{R}_{\text {in }}$ and $\tau_{\mathrm{m}}$ of the reduced and detailed models are essentially identical, the incomplete matching of early time constants inherent in the reduction method causes divergence in the input-output functions of the two models when complex, nonlinear active conductances are added to the soma and dendrites. Therefore, it is not possible to directly translate all the parameters used to model active conductances in the reduced model to those that would be used in more detailed models. However, many of the parameters used in compartmental models are obtained from the models themselves through the reiterative fitting process. Very few parameters are known with any certainty from actual measurements of real neurons. Parameters are usually employed that make the compartmental model behave in a manner similar to what is known about the behavior of the real neuron it represents. If it is realized that parameters used in the detailed models are neither unique nor certain, it is justifiable to allow the parameters used in the reduced models to be independent of those in the detailed model. If the major interest in a large-scale network is to examine the network interactions among realistically firing neuron models and not in the specific range of parameters used to create such behavior, then the use of reduced models is appropriate.

The question may be asked that if the particular parameters used in the model do not matter so long as the input-output function of the model is realistic, then why go through all the trouble of reducing a detailed cell model. Why not just create a generic cell prototype and constrain it to behave properly? Our answer to this question is straightforward. While the active properties of the reduced and detailed models are sometimes dissimilar, the passive properties of the two models are very similar and the method to generate the realistic cell prototype directly follows from the reconstructed morphology. Bcause $\mathrm{R}_{\text {in }}$ and $\tau_{\mathrm{m}}$ are two of the few properties that can be directly measured in real neurons, the close matching of these two properties by both the detailed and reduced models allow some direct comparisons to be 
A. B.
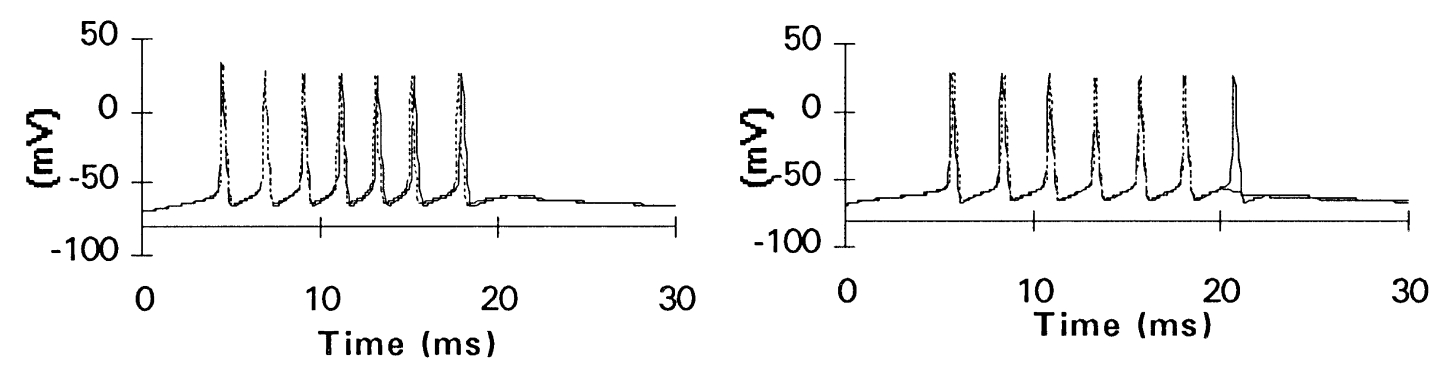

C.

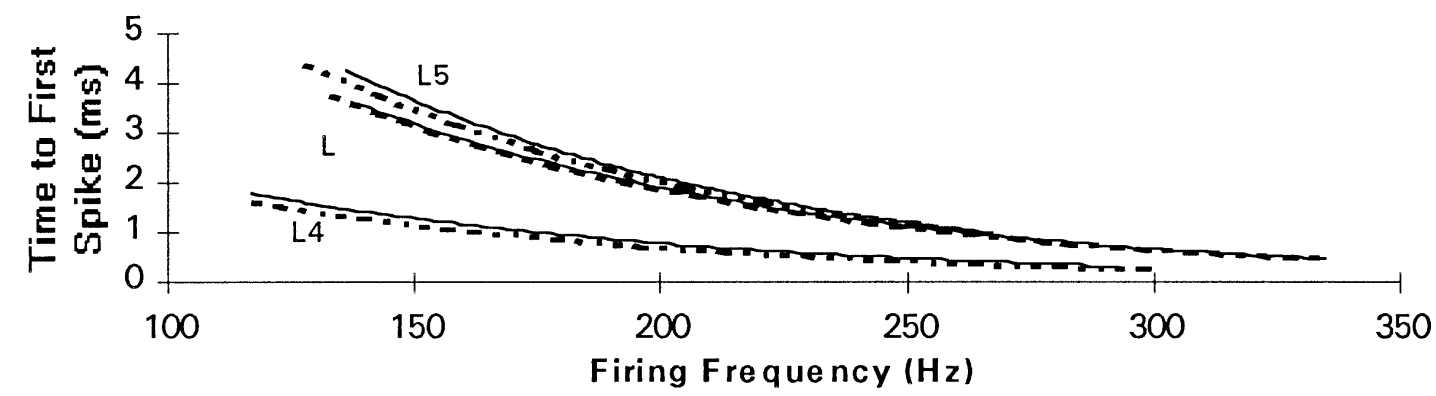

FIG. 6. (A) Somatic spike activity recorded in layer V cell models following constant $1 \mathrm{nA}$ current injection from $0 \mathrm{~ms}$ to $20 \mathrm{~ms}$. Active somatic kinetics were modeled as Hodgkin-Huxley type sodium and potassium channels. Parameters were $\mathrm{G}_{\mathrm{na}}=5.0 \mathrm{nS}, \mathrm{G}_{\mathrm{k}}=0.5 \mathrm{nS}$, and $\mathrm{T}=25^{\circ} \mathrm{C}$. The solid line is for the detailed compartmental model and the dashed line is for the reduced model. Note that the two traces are almost superimposed. (B) Same as in (A) except a $5 \mathrm{nA}$ current is injected into the most distal apical trunk segment. (C) Simulated input-output curves for each model with Hodgkin-Huxley type sodium and potassium channels plus A current. The firing frequency, calculated as the average of the first two interspike intervals, is plotted against the time to the first spike following a series of depolarizing current steps. The solid lines represent the detailed model and the dotted lines represent the reduced models.

made to the morphology of the real neuron. It is not known how much the dendritic morphology contributes to the behavior of a neuron, but it can potentially have a great influence on the behavior of a neuron by providing diverse pathways for synaptic integration as well as the contribution of individual time constants to the overall time constant of the membrane. However, the presence of active dendritic conductances [9,21] may eliminate the effects of variations in the dendritic morphology, creating essentially isopotential domains that serve as coincidence detectors rather than integrators [34]. Therefore, one of the advantages of the reduced models, in addition to their computational efficiency, is their reduced morphological complexity and their use can shed some light on the contribution of dendritic morphology to the behavior of the model. This is most easily done if both the reduced and the detailed compartmental models are both related to the same original morphology.

When constructing a biologically realistic neural network it is likely that one would wish to use neuron models that are not all identical to each other, but instead, each model should have some variation about some mean value. This would more closely represent the in vivo condition. There are several ways in which the modeler can produce such variation, including varying the morphology of each cell model, varying $R_{m}, C_{m}$, and $R_{a}$ to change $\mathbf{R}_{\text {in }}$ and $\tau_{\mathrm{m}}$ around the observed mean values, changing the type of active channels and their distribution and density, and also by the location and strength of synaptic inputs. The modeler must have an appreciation for which parameters are most relevant to the differences between different classes of neocortical neurons (regular spiking - adapting, bursting, and fast spiking), and which parameters are most important to individual neuron differences within the same class.

It is not entirely clear to what extent the defining response properties of the three major classes of neocortical cells (regular spiking, bursting, and fast spiking) depend on the electrotonic properties of the cells. Although McCormick et al. [26] found that neocortical bursting cells tend to have lower $\mathrm{R}_{\text {in }}$ and shorter $\tau_{\mathrm{m}}$ than regular spiking cells, and fast spiking cells tend to have even higher $\mathrm{R}_{\mathrm{in}}$ and shorter $\tau_{\mathrm{m}}$ than the two other cell classes, the differences between cell classes in their study were not significant, although Kasper et al. [18-20] did report significant differences in $\mathrm{R}_{\mathrm{in}}$ and $\tau_{\mathrm{m}}$ between regular spiking and bursting pyramidal neurons in layer $\mathrm{V}$ of rat visual cortex. In several studies that report $\mathbf{R}_{\mathrm{in}}$ and $\tau_{\mathrm{m}}$ for several cells of the same cell class, the measurements of $\mathbf{R}_{\mathrm{in}}$ and $\tau_{\mathrm{m}}$ are seen to vary as much as $50 \%$ from the mean value $[1,5,9,12,25,26]$. We found in the course of our studies that changing $\mathrm{R}_{\mathrm{m}}, \mathrm{R}_{\mathrm{a}}$, and $\mathrm{C}_{\mathrm{m}}$ by as much as $50 \%$ of their original value had little effect on the spike train produced by either the full or the reduced model in response to current injection. The timing of each spike certainly changes as $R_{\text {in }}$ and $\tau_{\mathrm{m}}$ change, but the regular spiking cell still produced a typical pattern of adapting spikes and the bursting cell still produced typical bursts of spikes. Changing $\mathrm{C}_{\mathrm{m}}$ has the least effect, only changing $\tau_{\mathrm{m}}$, which causes each spike to occur proportionately sooner or later. Changing $\mathrm{R}_{\mathrm{a}}$ effects both $\mathrm{R}_{\mathrm{in}}$ and the length con- 
stant, but because $R_{a}$ is usually a couple of orders of magnitude less than $R_{m}$, the effect on the timing of the spike train is relatively minor. Changing $R_{m}$ has the greatest effect on the timing of spikes in response to a continuous current injection, because changing $R_{m}$ changes $R_{i n}, \tau_{m}$, and the length constant. Randomly varying $R_{m}, C_{m}$, or $R_{a}$, either collectively or in isolation, can produce cell models that exhibit some variance in $\mathrm{R}_{\mathrm{in}}$ and $\tau_{\mathrm{m}}$ but which still produce spike trains appropriate to their cell class. It should be noted that the changes seen in the electrotonic properties are linear in relation to changes in the membrane parameters and are of similar magnitude between the full and the reduced cell models.

In several studies of neocortical neurons the clearest distinction between the major cell classes are their gross morphological characteristics. Kasper et al. [18-20] found that bursting cells in layer $\mathrm{V}$ of rat visual cortex were always pyramidal cells with thick apical trunks that terminated in extensive apical tufts, whereas regular spiking cells in the same layer were pyramidal cells with thin apical trunks and almost no apical tuft. Other studies identified regular spiking cells as pyramidal cells in the upper cortical layers with extensive apical tufts and deep layer pyramidal cells without apical tufts, identified bursting cells as large pyramidal cells in cortical layer $\mathrm{V}$ with extensive apical tufts, and identified fast spiking cells as small stellate cells located in all cortical layers II-VI [1,26]. The reduced models retain these gross morphological characteristics of the cell class upon which they are based, as seen in Fig. 1. The reduced bursting cell model (L5) retains the characteristic basal dendrite region, thick apical trunk, proximal and distal oblique apical dendrites, and extensive apical tuft. The reduced regular spiking cell model (L3) retains the basal dendrite region, relatively thin apical trunk, and extensive apical tuft typical of layer III pyramidal neurons. The reduced fast spiking cell model (L4) is modeled with only one
TABLE 1

TIME REQUIRED TO SIMULATE 1 SECOND ON A SPARC 20

\begin{tabular}{|c|c|c|}
\hline & Passive Model & Active Model \\
\hline Cell Model & $\begin{array}{l}\text { Full(s)/Reduced(s) } \\
\text { (Efficiency Increase) }\end{array}$ & $\begin{array}{l}\text { Full(s)/Reduced(s) } \\
\text { (Efficiency Increase) }\end{array}$ \\
\hline L3 & $\begin{array}{c}380 / 20 \\
(19 \times)\end{array}$ & $\begin{array}{l}2760 / 120 \\
(23 \times)\end{array}$ \\
\hline L4 & $\begin{array}{c}120 / 10 \\
(12 \times)\end{array}$ & $\begin{array}{l}750 / 60 \\
(12.5 \times)\end{array}$ \\
\hline L5 & $\begin{array}{l}80 / 4 \\
(20 \times)\end{array}$ & $\begin{array}{c}510 / 27 \\
(18.9 \times)\end{array}$ \\
\hline
\end{tabular}

dendritic region and without a prominent apical trunk. Although we did not model a layer $\mathrm{V}$ regular spiking cell similar to that described in Kasper et al. [20], it is clear that a reduced model of such a cell would reflect the general morphology of such a cell and have a thin apical trunk with a very limited apical tuft.

Although the electrotonic and morphological properties of the neuron are important contributors to the neurons response properties, the most important distinction between different cell types is likely to be the type and distribution of active conductances. Evidence is accumulating that a wide variety of voltage and ion dependent channels are located not only in the soma of neocortical neurons, but are also located throughout the dendrites and play a prominent role in the response properties of the neuron $[2,21,28,40]$. The bursting and adapting behavior typical of neocortical pyramidal neurons is closely linked to calcium dependent ion channels [41]. Physiological evidence exists that there are differences in the type and density of channels between the

A.

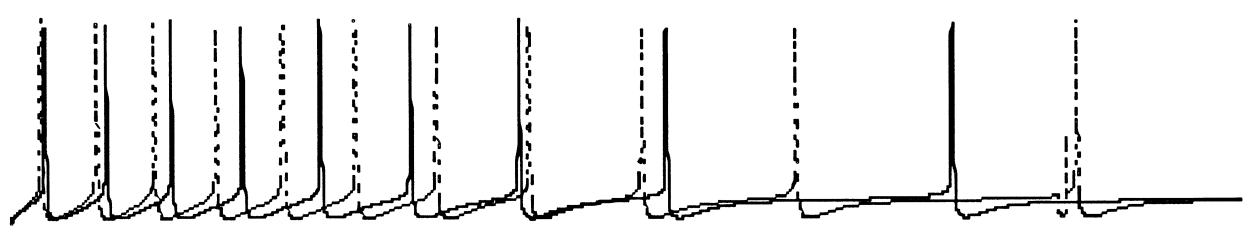

B.

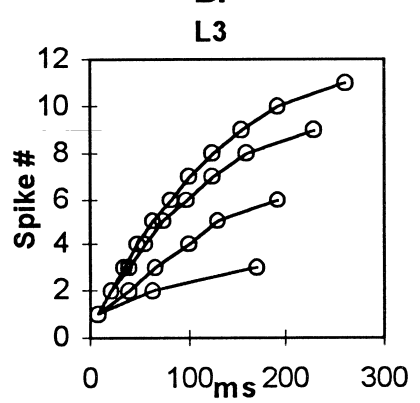

C.

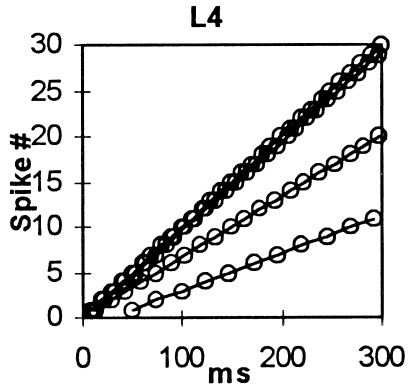

D.

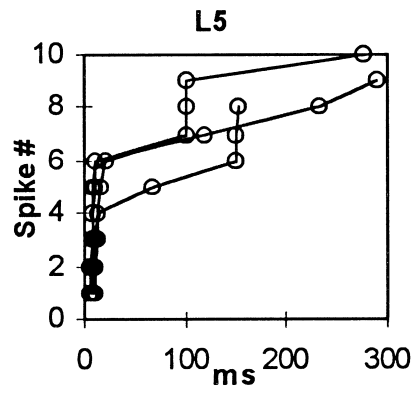

FIG. 7. (A) Adapting spike trains produced by the full (solid line) and reduced (dashed line) L3 models. Spike trains are similar but not well matched because of the cumulative effect of the incomplete matching of early time constants by the reduced model. (B-D) Although the reduced models do not perfectly match spike trains produced by the full models using identical parameters, the reduced models are capable of producing representative input-output curves of the appropriate cell type. These figures show the response of each cell to steps of increasing current intensity. The serial number of each spike is plotted as a function of its time of occurrence after stimulus onset. These compare well to representative firing patterns seen in Agmon and Connors Fig. 2 [1]. 
proximal and distal dendrites [33], and some modeling studies stress the importance of proper distribution of calcium channels to create bursting behavior $[36,38,40]$, although other studies discount the effect of spatial distribution in bursting behavior [27,37]. Indeed, in the practice of creating computational models of such neurons the proper selection of and density and distribution of such channels proves to be one of the most important parameters that the modeler must manipulate. Because our reduced models retain the general morphological characteristics of their respective neuron types, it is possible to explore the dimension of channel distribution between either proximal and distal dendritic regions or between basal and apical domains. As was mentioned before, reducing the dendritic tree into representative domains (i.e., basal, proximal oblique, distal oblique, and apical) offers the advantage of greatly reducing the parameter space needed to explore of the effects of dendritic conductances, and in many cases makes such an endeavor both possible and interpretable. Of course the limitation of such an exploration in a reduced model is that detailed examination of local effects within a dendritic domain is not possible.

Another parameter of neuron variability that is vitally important to network behavior is the strength, distribution, and type of synaptic input. Because the role of a neuron is to integrate synaptic inputs from other neurons and produce an appropriate synaptic output, synaptic integration is the most defining aspect of a neurons behavior. However, as seen in Fig. 5, the integration of dendritic synaptic inputs is the most severe limitation of the reduced models. Because, in the reduced model, all of the branches have been combined into a single equivalent cylinder, it is not possible to directly correlate synaptic inputs into a reduced dendritic section to identical inputs into an individual dendrite of a detailed model. It is also not possible to explore the spatial or temporal integration of synaptic inputs to different dendritic branches. This will greatly constrain the ability to explore the complex variety of synaptic interactions that probably occur in real neurons. Although it was seen in Fig. 5 that the reduced model cannot reproduce the full range of synaptic responses that are seen in the detailed model, the reduced model can produce synaptic responses that are within the appropriate range. Therefore, the reduced models can be used to explore the effects of a limited number of different synaptic inputs within the constraints of the above-mentioned limitations. Of course, in many cases, this can again be considered an advantage of reduced models as the possible parameter space is reduced, perhaps making the modeling results much easier to interpret. In addition, although the amount of synaptic integration is limited, using reduced computational models is certainly more biologically realistic than using artificial point neurons to study network behavior where the temporal dynamics are important.

We chose to create our reduced models for this paper using the method of Stratford et al. [35]. We also created reduced models using the method of Bush and Sejnowski [7] and found them to be essentially equivalent to our first reduced models. Both methods suffer the same limitations, and the choice of which method to use is probably one of personal preference. A future direction of our research will be looking at networks using both detailed and reduced models and determining if the reduced models are capable of the same network activity as the detailed models, and to that end we have used reduced models to explore the dynamic behavior of a small reciprocally connected network [17]. We hope to determine if detailed dendritic morphology is necessary or if a gross representation of that morphology is sufficient. We conclude that reduced models made using either method are appropriate for use in biologically realistic networks as long as the above-mentioned limitations are realized.

\section{ACKNOWLEDGEMENTS}

The authors wish to thank Barry Connors and Isabella Bulthoff.

\section{REFERENCES}

1. Agmon, A.; Connors, B. W. Correlation between intrinsic firing patterns and thalamocortical synaptic responses of neurons in mouse barrel cortex. J. Neurosci. 12:319-329; 1992.

2. Amitai, Y.; Friedman, A.; Connors, B. W.; Gutnick, M. J. Regenerative activity in apical dendrites of pyramidal cells in neocortex Cereb. Cortex. 3:26-38; 1993.

3. Bhalla, U. S.; Bower, J. M. Exploring parameter space in detailed single neuron models: Simulations of the mitral and granule cells of the olfactory bulb. J. Neurophysiol. 69:1948-1965; 1993.

4. Bower, J. M.; Beeman, D. The book of GENESIS. New York: Springer Verlag; 1995.

5. Brown, T. H.; Ficke, R. A.; Perkel, D. H. Passive electrical constants in three classes of hippocampal neurons. J. Neurophysiol. 46:812827; 1981 .

6. Brown, T. H.; Zador, A.; Mainen, Z. F.; Claiborne, B. J. Hebbian computations in hippocampal dendrites and spines. In: McKenna, T.; Davis, J.; Zornetzer, S. F., eds. Single neuron computation. San Diego: Academic Press; 1992:81-116.

7. Bush, P. C.; Sejnowski, T. J. Reduced compartmental models of neocortical pyramidal cells. J. Neurosci. Methods 46:159-166; 1993.

8. Cauller, L. J.; Connors, B. W. Functions of very distal synapses: Experimental and computational studies of layer I synapses on neocortical pyramidal cells. In: McKenna, T.; Davis, J.; Zornetzer, S. F., eds. Single neuron computation. San Diego: Academic Press; 1992:199-229.

9. Cauller, L. J.; Connors, B. W. Synaptic physiology of horizontal afferents to layer I in slices of rat SI neocortex. J. Neurosci. 14:751762; 1994.

10. Cauller, L. J.; Kulics, A. T. The neural basis of the behaviorally relevant N1 component of the somatosensory-evoked potential in SI cortex of awake monkeys: Evidence that backward cortical projections signal conscious touch sensation. Exp. Brain Res. 84:607-619; 1991.

11. Cole, K. S. Membranes, Ions and Impulses. Berkeley, CA: University of California Press 1968 .

12. Connors, B. W.; Gutnick, M. J.; Prince, D. A. Electrophysiological properties of neocortical neurons in vitro. J. Neurophysiol. 48:13021320; 1982.(Abstract)

13. Hines, M. A program for simulation of nerve equations with branching geometries. Int. J. Biomed. Comput. 24:55-68; 1989.

14. Holmes, W. R.; Rall, W. Estimating the electrotonic nature of neurons with compartmental models. J. Neurophysiol. 68:1438-1452; 1992.

15. Jack, J. J. B.; Noble, D.; Tsien, R. W. Electric current flow in excitable cells. Oxford: Calderon Press; 1975.

16. Jackson, M. E.; Cauller, L. J. Simplified computational models of neocortical neurons for use in anatomically realistic network simulations of interareal cortical oscillations. Soc. Neurosci. Abstr. 19:1993.

17. Jackson, M. E.; Patterson, J.; Cauller, L. J. Dynamical analysis of spike trains in a simulation of reciprocally connected "chaoscillators": Dependence of spike train fractal dimension on strength of feedback connections. In: Bower, J. M., ed. Computational neuoscience. San Diego: Academic Press; 1996:209-214.

18. Kasper, E. M.; Larkman, A. U.; Lubke, J.; Blakemore, C. Pyramidal neurons in layer 5 of the rat visual cortex. I. Correlation among cell morphology, intrinsic electrophysiological properties, and axon targets. J. Comp. Neurol. 339:459-474; 1994.

19. Kasper, E. M.; Larkman, A. U.; Lubke, J.; Blakemore, C. Pyramidal neurons in layer 5 of the rat visual cortex. II. Development of electrophysiological properties. J. Comp. Neurol. 339:475-494; 1994.

20. Kasper, E. M.; Lubke, J.; Larkman, A. U.; Blakemore, C. Pyramidal neurons in layer 5 of the rat visual cortex. III. Differential maturation 
of axon targeting, dendritic morphology, and electrophysiological properties. J. Comp. Neurol. 339:495-518; 1994.

21. Kim, H. G.; Connors, B. W. Apical dendrites of the neocortex: Correlation between sodium-and calcium-dependent spiking and pyramidal cell morphology. J. Neurosci. 13:5301-5311; 1993.

22. Koch, C.; Zador, A. The function of dendritic spines: Devices subserving biochemical rather than electrical compartmentalization. J. Neurosci. 13:413-422; 1993.

23. Larkman, A. U. Dendritic morphology of pyramidal neurones of the visual cortex of the rat: III. Spine distributions. J. Comp. Neurol. 306:332-343; 1991.

24. Larkman, A. U. Dendritic morphology of pyramidal neurones of the visual cortex of the rat: I. Branching patterns. J. Comp. Neurol. 306:307-319; 1991.

25. Mason, A.; Nicoll, A.; Stratford, K. Synaptic transmission between individual pyramidal neurons of the rat visual cortex in vitro. J. Neurosci. 11:72-84; 1991.

26. McCormick, D. A.; Connors, B. W.; Lighthall, J. W.; Prince, D. A Comparative electrophysiology of pyramidal and sparsely spiny stellate neurons of the neocortex. J. Neurophysiol. 54:782-806; 1985.

27. Migliore, M.; Cook, E. P.; Jaffe, D. B.; Turner, D. A.; Johnston, D. Computer simulations of morphologically reconstructed CA3 hippocampal neurons. J. Neurophysiol. 73:1157-1168; 1995.

28. Mills, L. R.; Niesen, C. E.; So, A. P.; Carlen, P. L.; Spigelman, I.; Jones, O. T. N-type $\mathrm{Ca}^{2+}$ channels are located on somata, dendrites, and a subpopulation of dendritic spines on live hippocampal pyramidal neurons. J. Neurosci. 14:6815-6824; 1994.

29. Rall, W. Branching dendritic trees and motoneuron membrane resistivity. Exp. Neurol. 1:491-527; 1959.

30. Rall, W. Theory of physiological properties of dendrites. Ann. NY Acad. Sci. 96:1071-1092; 1962.

31. Rall, W.; Burke, R. E.; Holmes, W. R.; Jack, J. J. B.; Redman, S. J. Matching dendritic neuron models to experimental data. Physiol. Rev. 72:s159-s186; 1992
32. Rall, W.; Rinzel, J. Branch input resistance and steady state attenuation for input to one branch of a dendritic neuron model. Biophys. J. 13:648-688; 1973.

33. Reuveni, I.; Friedman, A.; Amitai, Y.; Gutnick, M. J. Stepwise repolarization from $\mathrm{Ca}^{2+}$ plateaus in neocortical pyramidal cells: Evidence for nonhomogeneous distribution of HVA $\mathrm{Ca}^{2+}$ channels in dendrites. J. Neurosci. 13:4609-4621; 1993.

34. Softky, W. R.; Koch, C. The highly irregular firing of cortical cells is inconsistent with temporal integration of random EPSPs. J. Neurosci. 13:334-350; 1993.

35. Stratford, K.; Mason, A.; Larkman, A. U.; Major, G.; Jack, J. J. B. The modeling of pyramidal neurones in the visual cortex. In: Durbin, R.; Maill, C.; Mitchison, G., eds. The computing neuron. Reading, MA: Addison-Wesley. 1989:296-321.

36. Traub, R. D. Neocortical pyramidal cells: A model with dendritic calcium conductance reproduces repetitive firing and epileptic behavior. Brain Res. 173:243-257; 1979.

37. Traub, R. D. Simulation of intrinsic bursting in CA3 hippocampal neurons. Neuroscience 7:1233-1242; 1982.

38. Traub, R. D.; Jefferys, J. G.; Miles, R.; Whittington, M. A.; Toth, $\mathrm{K}$. A branching dendritic model of a rodent CA3 pyramidal neurone. J. Physiol. (Lond.) 481:79-95; 1994.

39. Traub, R. D.; Miles, R. Model of the origin of rhythmic population oscillations in the hippocampal slice. Science 243:1319-1325; 1989.

40. Traub, R. D.; Wong, R. K.; Miles, R.; Michelson, H. A model of a CA3 hippocampal pyramidal neuron incorporating voltage-clamp data on intrinsic conductances. J. Neurophysiol. 66:635-650; 1991.

41. Warman, E. N.; Durand, D. M.; Yuen, G. L. Reconstruction of hippocampal CA1 pyramidal cell electrophysiology by computer simulation. J. Neurophysiol. 71:2033-2045; 1994.

42. Wilson, M.; Bower, J. M. Cortical oscillations and temporal interactions in a computer simulation of the piriform cortex. J. Neurophysiol. 67:981-995; 1992 